\title{
Rheological response of nonspherical granular flows down an incline
}

\author{
R. C. Hidalgo, ${ }^{1, *}$ B. Szabó, ${ }^{2}$ K. Gillemot, ${ }^{2}$ T. Börzsönyi, ${ }^{2, \dagger}$ and T. Weinhart ${ }^{3, \ddagger}$ \\ ${ }^{1}$ Departamento de Física y Matemática Aplicada, Facultad de Ciencias, Universidad de Navarra, \\ Navarra, Spain \\ ${ }^{2}$ Institute for Solid State Physics and Optics, Wigner Research Centre for Physics, \\ Hungarian Academy of Sciences, P.O. Box 49, H-1525 Budapest, Hungary \\ ${ }^{3}$ Multiscale Mechanics, University of Twente, P.O. Box 217, 7500 AE Enschede, Netherlands
}

(Received 9 November 2017; published 3 July 2018)

\begin{abstract}
We present an extensive numerical and experimental study, investigating a threedimensional (3D) granular flow of elongated particles down an inclined plane. Similarly to sheared systems, the average particle orientation is found to enclose a small angle with the flow direction. In the bulk, this behavior is independent of the shear rate. At the surface, however, the particles move in more dilute conditions, and the average orientation strongly depends on the shear rate. A systematic numerical study varying the particle aspect ratio and the plane inclination reveals that the particle size perpendicular to the flow direction, $d_{\text {eff }}$, is an appropriate length scale to define an effective inertial number $I_{\text {eff }}$, which fully captures the impact of the particle shape on the system's rheology. Like in the case of spheres, density and friction result in well-defined functions of the effective inertial number $I_{\text {eff }}$. Thus, we quantify and explain the dependence of the rheological parameters on the aspect ratio, based on the micromechanical details.
\end{abstract}

DOI: 10.1103/PhysRevFluids.3.074301

\section{INTRODUCTION}

Despite its scientific and technological significance, a general theoretical framework to describe the rheological response of granular systems is still lacking. However, several promising approaches have recently been published [1-6]. In particular, it is known that various modes of local energy dissipation, such as mechanical friction or plastic deformation, may lead to nonequilibrium steadystate situations.

Examining granular flows of spherical particles experimentally and numerically, the $\mu(I)$ rheology is successfully used in dense, quasistatic, and inertial regimes [7-14]. This theory postulates that the macroscopic flow density $\rho(I)$ and bulk friction $\mu(I)$ are functions of the inertial number $I$, which is the ratio of two timescales, $I=\tau_{p} / \tau_{s}$, the pressure-induced inertial time $\tau_{p}$, and the inverse shear rate $\tau_{s}=\dot{\gamma}^{-1}$. Thus, quasistatic flows result in values $I \ll 1$, which denotes a much slower local deformation rate than a typical inertial relaxation. Exploring the behavior of granular flows, Da Cruz et al. [8] numerically numerically observed a linear increase of the bulk friction $\mu(I)$ with $I$ and a linear decrease of the bulk density $\rho(I)$, starting from the static internal friction value. However, Jop and coworkers [7] measured a nonlinear response when examining a system at moderate inertial

\footnotetext{
*raulcruz@unav.es

†borzsonyi.tamas@wigner.mta.hu

łt.weinhart@utwente.nl
} 
numbers, ranging from 0.01 to 0.5 . Moreover, Hatano [9] proposed that $\mu(I)$ increases as a power law, which agrees with Bagnold's scaling relations [15].

Although granular flows are often composed of nonspherical shapes, most of the research usually considers spherical particles [13-19]. However, it is known that particle shape may give rise to a different mechanical response and complex flow patterns [20-30]. Interestingly, the collective motion of elongated particles usually induces particles to orient themselves along a preferred direction, forming an angle with the streamlines [20-23]. This shear alignment seems to decrease the system's effective friction, compared to the nonordered state [22]. Moreover, its geometric origin makes it a robust phenomenon, which is even found at molecular length scales such as in nematic liquid crystals [31-33].

Discrete element method (DEM) simulations of nonspherical granular systems have recently received significant attention (see review in Ref. [34]). Implementing particle shape is computationally expensive. Therefore, to mimic its effects, simple rolling friction has been used in systems of spheres [35]. Other authors have reproduced particle shape, identifying it directly from digitized images [36], using polygons or polyhedra [37-40], superquadrics [41], spheropolygons or spheropolyhedra [42,43], nurbs [44], level sets [45], and clumps of disks or spheres [46-48]. Moreover, advanced models that include the contact geometry and particle geometry have been developed by combining DEM with finite element formulations [36,49].

Despite its significance for practical applications, a well-founded $\mu(I)$-rheological formulation for flows of nonspherical particles is still lacking. Recent numerical studies on two-and three-dimensional (2D and 3D) systems of frictional dumbbells yielded increasing effective friction with increasing particle elongation in chute flows, as well as in a Couette shear cell $[46,47]$. Another numerical study with frictionless spherocylinders in $3 \mathrm{D}$ resulted in a nonmonotonic but predominantly decreasing effective friction as a function of the particle aspect ratio in Couette shear [50,51].

In this paper, we present an extensive numerical and experimental study, investigating a frictional 3D granular flow of elongated particles down an inclined plane. Similarly to sheared systems, the average particle orientation is found to enclose a small angle with the flow direction. Interestingly, this is significant when explaining the rheological response of those systems. Using a mathematically consistent averaging technique, the numerical data reveal that the particle size perpendicular to the flow direction is an appropriate length scale to define an effective inertial number $I_{\text {eff }}$, introducing a generalized $\mu(I)$ rheological formalism. Additionally, our outcomes clearly indicate that the effective friction of an assembly of frictional 3D spherocylinders increases with aspect ratio. The paper is organized as follows: In Sec. II, we describe the experimental setup, in Sec. III, we mention the numerical model and its implementation, and Sec. IV includes the main results and its discussion. At the end, conclusions and outlook are presented.

\section{EXPERIMENTAL SYSTEM}

We examine experimentally granular flows on an inclined plane of length $2 \mathrm{~m}$ and width $0.4 \mathrm{~m}$ [Fig. 1(a)]. The surface of the plane is covered with coarse sandpaper (grit P40) to ensure uniform roughness. A hopper at the top of the plane provides grains at a uniform flow rate, independent of the plane inclination $\alpha$. The motion of the grains in the surface layer is recorded by two fast cameras (Mikrotron EoSens CL MC1362) using a frame rate of $1000 \mathrm{fps}$ and a spatial resolution of $10 \mathrm{pixel} / \mathrm{mm}$. Camera 1 records the $x y$ plane while camera 2 records the $x z^{\prime}$ plane, which is inclined at a small angle $\beta=17^{\circ} \pm 1^{\circ}$ with respect to the vertical $x z$ plane. We use cylindrical glass rods of diameter $d=1.9 \mathrm{~mm}$ and length-to-width ratio $\xi=L / d=3.5$. The granular sample contained $6 \%$ of black tracer particles. The image sequences taken by the two cameras are analyzed separately. In both cases, the orientation of the black tracer particles $\left(\theta^{\prime}\right.$ and $\left.\phi\right)$ with respect to the flow direction (see Fig. 1) is determined. This is done with a specially developed algorithm. We take advantage of the cylindrical grain shape, where the two-dimensional projection of a particle consists of two nearly parallel sides. After the algorithm detects the appropriate sized black areas on an image, it calculates the direction of the gradient around the particle contour. For a clearly visible 


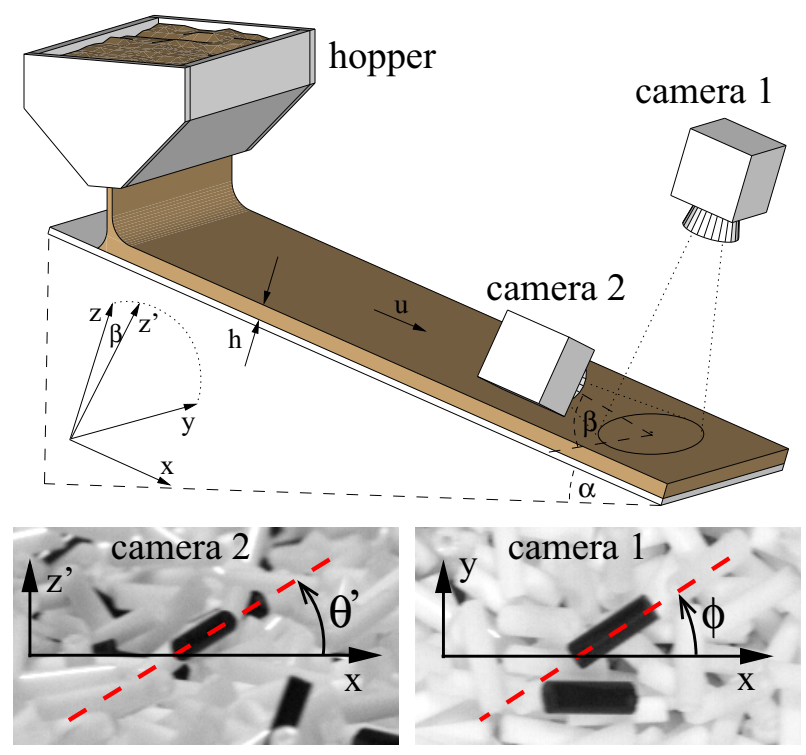

FIG. 1. Sketch of the experimental setup. Dimensions of the inclined plane: $2 \mathrm{~m} \times 0.4 \mathrm{~m}$. Granular material: glass rods with length of $6.6 \mathrm{~mm}$ and diameter of $1.9 \mathrm{~mm}$. Sample images taken with cameras 1 and 2 and the definitions of the two angles $\left(\theta^{\prime}\right.$ and $\left.\phi\right)$ are shown.

grain, the distribution of these orientations consists of a high and sharp peak perpendicular to the particle orientation (corresponding to the long side) and a second peak parallel to it (corresponding to the ends). If the grain is fully visible, or only slightly hidden by an overlapping white particle, the method easily determines the grain orientation. If the overlap is larger, the method recognizes this in the distribution of the gradients. In the current analysis, we do not consider grains where the second largest peak is outside the range of $90^{\circ} \pm 2^{\circ}$ with respect to the main peak.

\section{NUMERICAL MODEL}

Numerically, a chute flow of spherocylinders is modeled using a hybrid GPU-CPU discrete element algorithm (DEM) [42,52]. The aspect ratio of the spherocylinders is defined by $\xi=L / d=(l+$ $2 r) / 2 r$, where $l$ is the cylinder length and $r$ is the spheroradius. The chute is inclined at an angle $\alpha$ such that gravity acts in the direction $[\sin (\alpha), 0,-\cos (\alpha)]$. The size of the simulation cell was $S_{x}=S_{y}=16 L$ and $S_{z}=32 L$, and periodic boundary conditions were used in the $x$ and $y$ directions; i.e., after leaving one of the boundaries, the particles are put back at the other with the same velocity and inclination.

Initially, $N_{p}$ particles are inserted into the simulation cell at a random location and with random orientation. In each case, the number of particles $N_{p}$ is adjusted to the choice of $\xi$ such that the height of the flow in steady-state conditions is roughly $H=20 \mathrm{~L}$.

The equation of motion of particle $i(i=1, \ldots, N)$ reads as

$$
\sum_{j=1}^{N_{c}} \vec{F}_{i j}+m \vec{g}=\vec{F}_{i}=m \vec{a}_{i}
$$


for the translational degrees of freedom. Complementarily, Euler's equations describe the rotational motion,

$$
\sum_{j=1}^{N_{c}} \vec{\tau}_{i j}=\vec{M}_{i}=I \overrightarrow{\dot{\omega}}+\vec{\omega} \times(I \vec{\omega}),
$$

where $m$ is the mass of the particle and $I$ is the moment of inertia tensor. $\vec{F}_{i j}$ is the force exerted by particle $j$ on particle $i$, and $\tau_{i j}$ accounts for its corresponding torque. The total force $\vec{F}_{i}$ and momentum $\vec{M}_{i}$ acting on particle $i$ are obtained as sums of the pairwise interaction of particle $i$ with its contacting neighbors.

The translational equations of motion are integrated using a velocity Verlet numerical algorithm implemented as CUDA-thrust functions, which are inside the DEM loop. The set of Eqs. (2) defines the particle's angular velocity $\vec{\omega}$, in the body frame. Additional equations are necessary to compute the particle orientation. We adopted the quaternion representation and the rotational equations of motion are integrated using a leap-frog numerical algorithm implemented as CUDA-thrust functions (see details in Ref. [42]).

In our numerical approach, the contacting force between particles $i$ and $j$ has a normal and a tangential component $\vec{F}_{i j}=\vec{F}_{i j}^{n}+\vec{F}_{i j}^{t}$. The normal force $\vec{F}_{i j}^{n}$ depends linearly on the particles' overlap $\delta$. Moreover, the normal dissipation is proportional to the normal relative velocity $\vec{v}_{\text {rel }}^{n}$. Hence, the total normal force reads as $\vec{F}_{i j}^{n}=-k_{n} \delta \hat{n}-\gamma_{n} \vec{v}_{\text {rel }}^{n}$, where $k_{n}$ and $\gamma_{n}$ represent elastic and damping coefficients. $F_{i j}^{t}$ also includes elastic and viscous contributions, $\vec{F}_{i j}^{t}=-k_{t} \vec{\xi}-\gamma_{t} \vec{v}_{\text {rel }}^{t}$, where $\gamma_{t}$ is a damping coefficient and $\vec{v}_{\text {rel }}^{t}$ is the tangential relative velocity of the overlapping pair. $|\vec{\xi}|$ represents the elongation of an imaginary spring with elastic constant $k_{t}$. As long as there is an overlap between $i$ and $j, \vec{\xi}$ increases as $d \vec{\xi} / d t=\vec{v}_{\text {rel }}^{t}$ [53]. The elastic tangential elongation $\vec{\xi}$ is kept orthogonal to the normal vector and it is truncated as necessary to satisfy the Coulomb constraint $\left|\vec{F}_{i j}^{t}\right|<\mu_{p}\left|\vec{F}_{i j}^{n}\right|$.

The majority of the numerical results presented here correspond to glass beads; in the simulation, we use $k_{t}=\frac{2}{7} k_{n}, \gamma_{t}=\gamma_{n}, e_{n}=0.88, \rho_{p}=2500 \mathrm{~kg} / \mathrm{m}^{3}$, and $k_{n}=2 \times 10^{5}\left(m_{p} g / l\right)[13,16]$. The microscopic friction coefficient is set to $\mu_{p}=0.5$ and $\mu_{p}=0.8$ (rough particles). To compare with the experimental results, we set gravity to $g=10 \mathrm{~m} / \mathrm{s}^{2}$ and executing a systematic study of the system response we used $g=1 \mathrm{~m} / \mathrm{s}^{2}$. Moreover, in some specific cases we have simulated steel beads with $\rho_{p}=7850 \mathrm{~kg} / \mathrm{m}^{3}$.

DEM provides the forces experienced by each grain and also tracks their velocities and positions. From this data, the macroscopic mass density $\rho(\vec{r}, t)$ and velocity $\vec{V}(\vec{r}, t)$ fields are computed using a coarse-graining methodology [54,55]. Here, we use a truncated Gaussian coarse-graining function $\phi(\vec{r})=A_{w}^{-1} e^{-(|\vec{r}| / 2 w)^{2}}$ with cutoff $r_{c}=6 w$ where the value of $w=l / 4$ defines the coarse-grained scale. $A_{w}$ is numerically calculated so that the normalization condition holds. The data are confirmed to be homogeneous in $x, y$, and $t$, and therefore averaged over these dimensions. The velocity field in the flow direction, $V_{x}(z)$, is then used to calculate the macroscopic shear rate $\dot{\gamma}_{x}(z)$ by central finite differencing. Additionally, we compute the macroscopic stress tensor $\sigma_{\alpha \beta}$, which is split into a contact and kinetic stress contribution, $\sigma_{\alpha \beta}=\sigma_{\alpha \beta}^{c}+\sigma_{\alpha \beta}^{k} . \sigma_{\alpha \beta}^{c}$ is deduced using the relative position of the contacting particles, the contact point, and their interaction force, while $\sigma_{\alpha \beta}^{k}$ accounts for the velocity fluctuations of a single particle with respect to the macroscopic flow [54-56].

\section{RESULTS AND DISCUSSION}

\section{A. Steady-state flow}

In the experimental system, the hopper feeds the grains at a constant flow rate. Thus, after a short transient, a steady-state granular flow with a given height establishes on the inclined plane. Figure 2 shows the surface velocity as a function of time obtained by PIV analysis for $\alpha=26^{\circ}$ and $\alpha=33^{\circ}$ at two locations $x=158 \mathrm{~cm}$ and $x=165 \mathrm{~cm}$ below the hopper. This figure demonstrates that at 


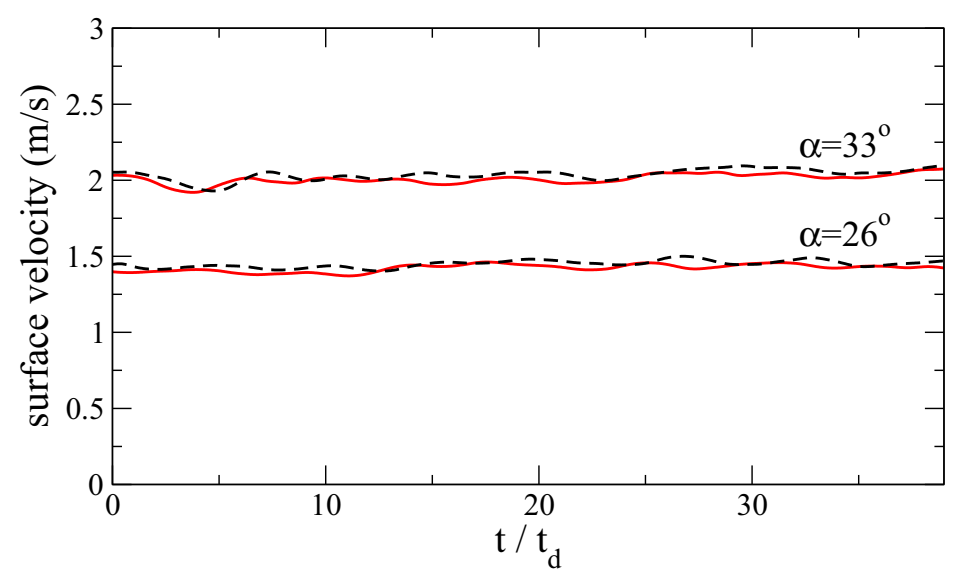

FIG. 2. The surface velocity from laboratory measurements for $\alpha=26^{\circ}$ and $\alpha=33^{\circ}$ taken at two locations $x=158 \mathrm{~cm}$ (continuous lines) and $x=165 \mathrm{~cm}$ (dashed lines) below the hopper.

the location where the data were taken the flow essentially does not accelerate any more, even for the highest inclination angle. Note that the steady-state momentum balance requires that the stress divergence and gravitational forces are in balance [13],

$$
\partial \sigma_{x z} / \partial z=-\rho g_{x},
$$

and thus

$$
\mu=-\sigma_{x z} / \sigma_{z z}=\tan (\alpha)
$$

where $\mu$ is known as a basal friction coefficient.

The simulations are executed until the system's kinetic energy reaches a constant value, i.e., when the ratio of mean kinetic energy and the mean elastic potential energy becomes time independent [13], reproducing the experimental conditions. For all particle elongations, the run time was at least $t=2000 \times t_{d}$, where $t_{d}=\sqrt{L / g}$. After that period of time, we sample the system evolution each $\Delta t=t_{d} / 4 \mathrm{~s}$.

Given the micromechanical data of all particles, one captures entirely the steady-state kinetics using the coarse-graining methodology, described earlier in the text. For instance, computing the averaged stress fields $\bar{\sigma}_{x z}$ and $\bar{\sigma}_{z z}$ we validate the steady-state conditions, comparing with Eq. (4). Note that we obtain excellent accuracy for all the types of particles. Figure 3 illustrates results for a range of inclinations in which the flow slowly evolves to a steady state. For higher inclinations, no steady state is reached, and for lower inclinations, the flow quickly arrests.

\section{B. Flow morphology}

As discussed further below in the text, details of the flow morphology can be used for clarifying the system's rheological response. We study the flow morphology experimentally and numerically for particles with elongation $\xi=3.5$ and inclination angles of $\alpha \in\left[28.5^{\circ}, 32.5^{\circ}\right]$ (numerically) and $\alpha \in$ $\left[26.0^{\circ}, 33.0^{\circ}\right]$ (experimentally). First, we compare data obtained at the surface of the flow from experiments and numerical simulations. The average alignment angles $\theta_{\mathrm{av}}^{\prime}$ and $\phi_{\mathrm{av}}$ as a function of the plane inclination are shown in Fig. 4(a). As expected, when looking from above, the particles are in average aligned with the streamlines, i.e., $\phi_{\mathrm{av}}=0$, independent of the plane inclination. When looking from the side, however, the particles orient along a nonzero angle with respect to the streamlines. The angle $\theta_{\text {av }}$ has a small positive value for lower plane inclinations (denser flows), as was observed in sheared systems experimentally and numerically [20-23]. However, increasing plane inclination leads to a decreasing alignment angle, which even changes sign for large inclinations. Remarkably, this trend 


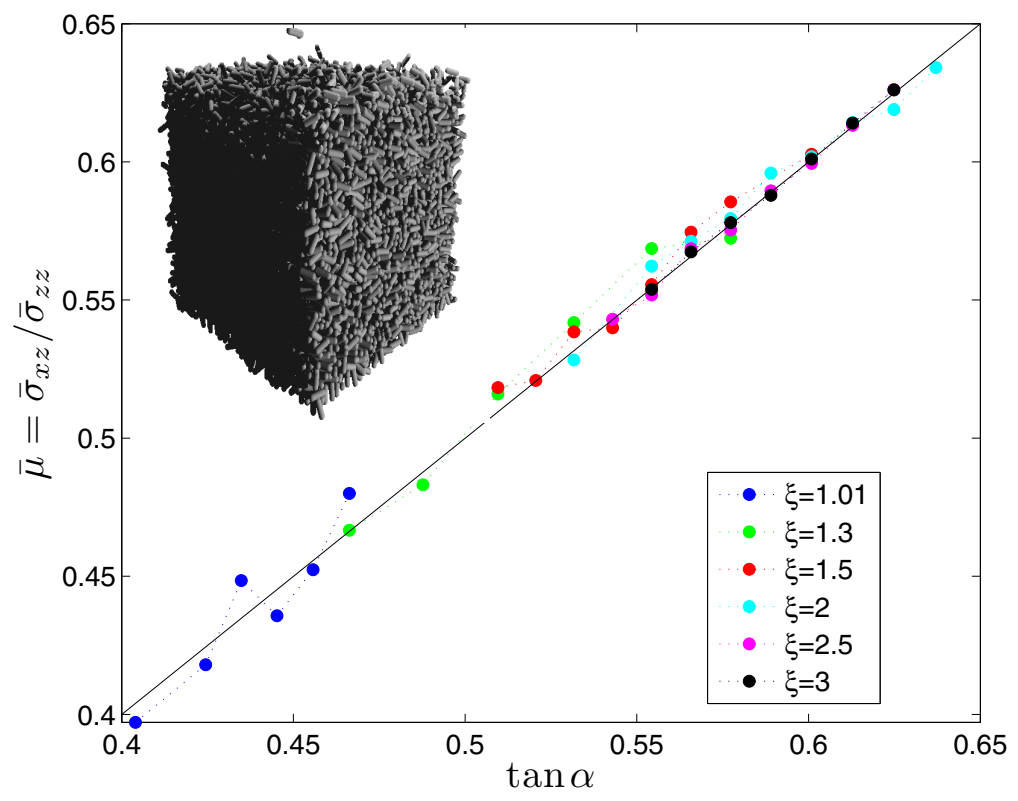

FIG. 3. Average friction $\bar{\mu}$ as a function of the plane inclination $\alpha$, obtained numerically, in steady-state conditions. Outcomes corresponding to glass particles, several-particle elongation $\xi$, and $g=1 \mathrm{~m} /{ }^{2}$ are shown. The inset illustrates the numerical system.

is similarly observed both in experiment and simulation. In the simulations, in the limit of low plane inclination the system is at rest and consequently the particle inclination angle should be zero.

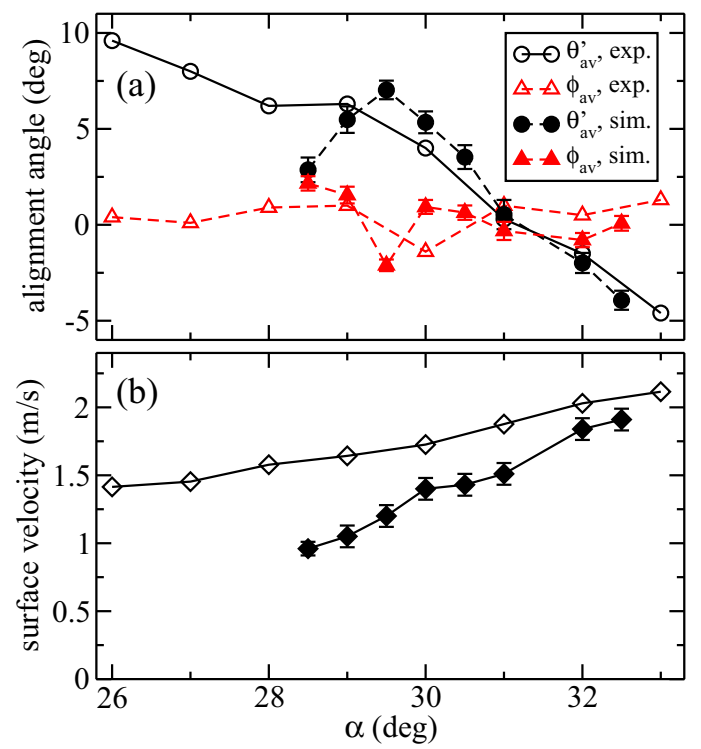

FIG. 4. (a) The average alignment angles $\theta_{\mathrm{av}}^{\prime}$ and $\phi_{\mathrm{av}}$ as a function of $\alpha$ at the surface from experiment (empty symbols) and simulation (full symbols). (b) The average surface velocity of the flow as a function of the plane inclination $\alpha$. Experimental data are shown with empty symbols, while the simulation data are indicated with full symbols. All data are obtained with glass particles of aspect ratio $\xi=3.5$. 


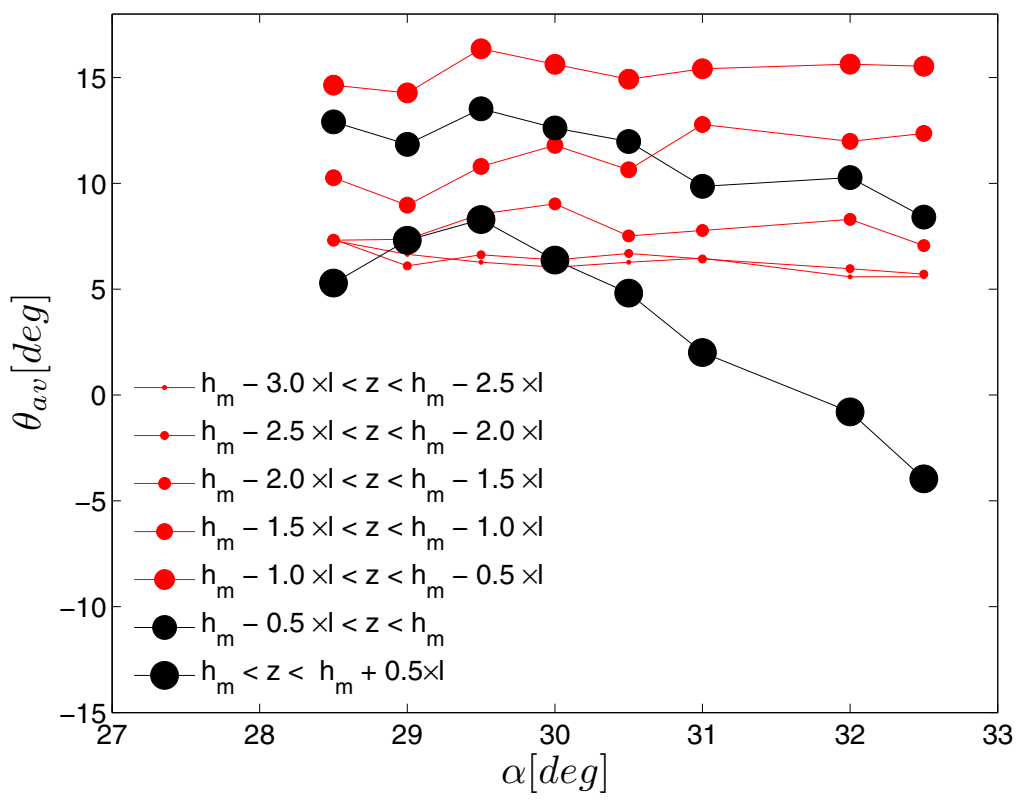

FIG. 5. The average alignment angles $\theta_{\text {av }}$ obtained numerically as a function of $\alpha$ for various layers. Red symbols indicate regions at $z<h_{m}$ and black symbols indicate those at $z>h_{m}$. The data correspond to glass particles and $g=10 \mathrm{~m} / \mathrm{s}^{2}$.

In Fig. 4(b), the surface velocity is shown as a function of the plane inclination. It is noticeable that the experimental and numerical data show similar trends: The velocity increases as the angle of inclination is increased. We argue that the small disagreement is understandable given that in the experimental system the particle volumetric flow rate is imposed. Accordingly, the height of the granular flow is different for each inclination angle. However, the simulation is done using a constant number of particles.

While the experimental data are limited to observations at the flow surface, DEM simulations allow access to the bulk statistics. Figure 5 shows the average alignment angle $\theta_{\text {av }}$ in different layers of the flow as a function of the plane inclination $\alpha$. The layers have a thickness of $\Delta z=L / 2$ and are located at a different distance from the mean surface height $h$. Black symbols represent regions at $z>h_{m}$, while red symbols stand for regions below the mean surface height. It is noticeable that the bulk behavior varies distinctly from the surface behavior, where the system is more dilute (more detailed density data are shown later in the text). While in the surface region $\theta_{\mathrm{av}}$ changes with height (above and below $h_{m}$ ) and the chute inclination $\alpha$ (above $h_{m}$ ), it is practically independent of $\alpha$ in the bulk region. Remarkably, the result in the bulk is in excellent agreement with observations in sheared systems [21-23], which also found that the alignment angle is independent of the velocity gradient $\dot{\gamma}$. Nevertheless, we observe that just above the mean surface, $\theta_{\mathrm{av}}$ increases with height, which correlates with a decrease of the velocity gradient $\dot{\gamma}$ (data not shown). Below the mean surface, however, $\theta_{\mathrm{av}}$ diminishes with increasing height and starts to depend on the plane inclination $\alpha$, which correlates with an abrupt increase of the velocity gradient $\dot{\gamma}$ (data shown later in the text). Similar trends were obtained analytically [33] and experimentally [32] at molecular length scales for a single Brownian rod subjected to shear flow. It is important to remark that higher inclination angles lead to larger momentum transfer and, consequently, when a particle leaves the surface it can reach a higher elevation where its orientation is less determined by the global flow. 


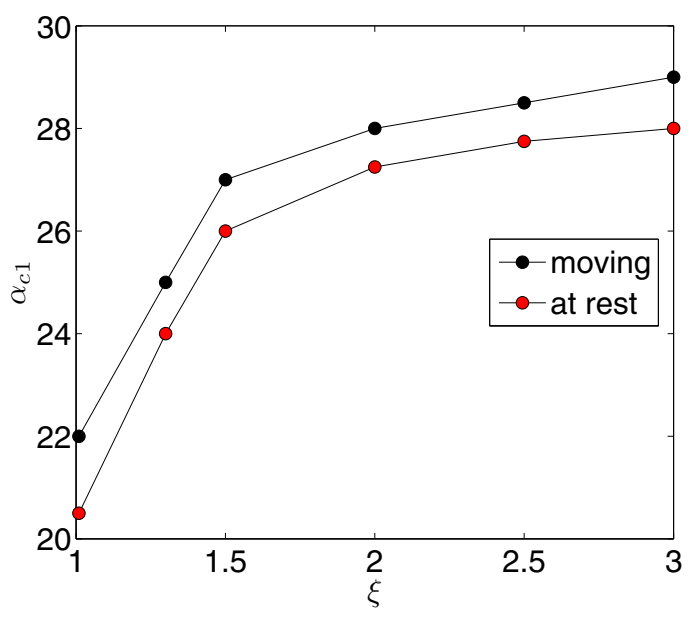

FIG. 6. Angle of repose $\alpha_{c 1}$ obtained numerically for glass particles with different elongations $\xi$ and $g=$ $10 \mathrm{~m} / \mathrm{s}^{2}$. Black markers show the highest angle at which the flow arrests, and red markers show the lowest angle at which steady flow is observed.

\section{Uniform steady flow of elongated particles}

In order to clarify the inter-relation between the flow morphology and the system's rheological behavior, a numerical study is performed, varying the particle aspect ratio $\xi$ and the plane inclination $\alpha$.

It is known that granular flows down inclined surfaces can be characterized by two critical angles. First, $\alpha_{c 1}$ is the angle at which the material comes to rest; for angles below, friction dominates over gravity. On the contrary, there is a value $\alpha_{c 2}$ above which gravity dominates over friction and the material accelerates. Between these two angles, steady flow is possible. In general, the exact values of $\alpha_{c 1}$ and $\alpha_{c 2}$ depend on polydispersity, particle shape, the basal surface, and the mean height of the bed. However, the location of the free surface of a granular flow, i.e., the exact flow height, is not well defined. For the sake of simplicity, simulations are computed using rods with $\xi \in[1.01,3.0]$, but always keeping the rod longer side $L$ and the total mass of particles. In each case, the values of $r$ and number of particles $N_{p}$ are adjusted to the choice of $\xi$. With these conditions, the height of the bed is roughly $H=20 \times L$ in all cases. The base of the system is a rough surface consisting of $N_{b}=385$ fixed spherical particles of radius $R=L / 2$. These conditions resemble one particular surface roughness of Ref. [57] and similar microscopic parameters.

First, we measured the angle of repose $\alpha_{c 1}$ and its dependence on $\xi$; the data obtained for particles with various $\xi$ are shown in Fig. 6 . For the sake of clarity, the plot illustrates the highest angle at which the flow arrests and the lowest angle in which the system is slightly moving. The data indicate that $\alpha_{c 1}$ increases with the particle elongation. This result is expected as nonsphericity is known to increase the stability of a static packing, leading to higher angles of repose [58-60]. However, it also noticeable that the rate of increase is reduced for aspect ratios above 1.5, in agreement with previous findings for rough ellipsoids [60].

As a second step, steady-state situations with $\alpha>\alpha_{c 1}$ for different $\xi$ are explored. Using the coarse-graining methodology, we calculate the macroscopic mass density $\rho(\vec{r}, t)(z)$ and velocity $\vec{V}(\vec{r}, t)(z)$ profiles. Because of symmetry reasons, those macroscopic profiles are uniform (data not shown) on the whole domain of $x$ and $y$ within our numerical accuracy. Moreover, we find that the mean velocities in the $y$ and $z$ directions are diminished as a result of the absence of forces in those directions. Figure 7 displays in detail the field profiles in the $z$ direction that are obtained for each particle elongation and several inclination angles. The density and velocity profiles [see Figs. 7(a) and $7(b)$ ] of these flows are very similar to those observed for spherical particles. The mass density 
(a)

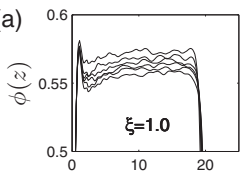

(b)

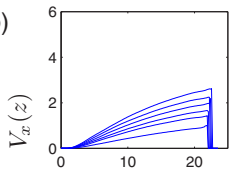

(c)

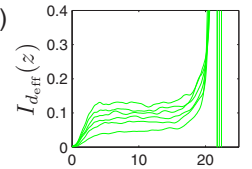

(d)

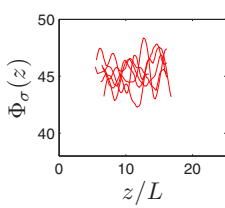

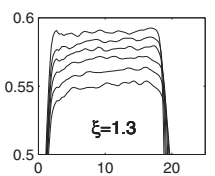
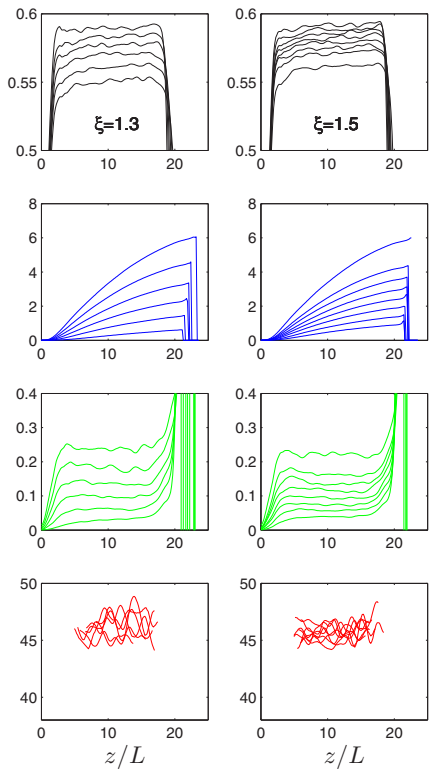
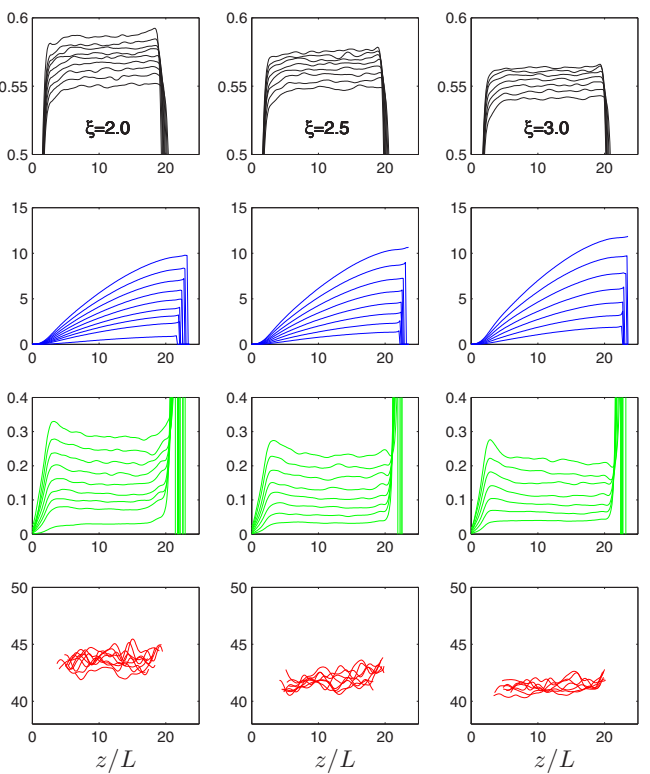

FIG. 7. Numerical results: the vertical profiles of volume fraction $\phi(z)$ (a), macroscopic velocity on the $x$ direction $V_{x}(z)(\mathrm{b})$, effective inertial number $I_{\mathrm{eff}}(z)(\mathrm{c})$, and angular stress eigendirection $\Phi_{\sigma}(z)$ (d). Results for glass particles and several particle elongations are shown, $\xi \in(1.01,3.0)$; in each figure, different lines correspond to different plane inclinations. Larger inclinations correspond to smaller volume fraction and larger velocity and effective inertial number values.

throughout the flow is practically constant in the bulk, decreasing slightly close to the base. Note that a constant density profile is a feature of all steady flows (varying $\alpha$ and $\xi$ ). Moreover, in all cases we obtain Bagnold-type velocity profiles $V_{x}(z)$ and the maximum velocity increases with increasing chute inclination.

Next, the values of $V_{x}(z)$, are used to calculate the macroscopic shear rate $\dot{\gamma}_{x}(z)$ by central finite differencing (data not shown). Moreover, their corresponding $I_{\text {eff }}(z)$ values are calculated via Eq. (5). The $I_{\text {eff }}(z)$ profiles obtained for several particle elongations and plane inclinations are illustrated in Fig. 7(c). As seen earlier with spherical particles, we find that the $I_{\text {eff }}(z)$ and $\rho(z)$ profiles are practically constant in the bulk region, with larger variations only in the basal and surface layers. The latter is a key assumption of depth averaging and it justifies the use of a $\mu(I)$-rheology scheme.

Interesting micromechanics features can be deduced from the eigenvectors of the mean stress tensor $\sigma_{\alpha \beta}$, which, for instance, allows us to compare the main plane of action of the stress and strain tensors. The idea is to clarify the impact of the particle shape on local stress transmission and its relation with the shear strain. Note that in plane strain conditions the eigenvector of the shear strain points to $\Phi_{\varepsilon}=45^{\circ}$. Similar to the case of spheres, we find that the $z$ components of the $n^{1}$ and $n^{3}$ eigenvectors nearly vanish, except for statistical fluctuations. Moreover, the $x$ and $y$ components of the second eigenvector $n^{2}$ also vanish. Then, the angular stress eigendirection was defined as $\Phi_{\sigma}=\tan ^{-1}\left(n_{y}^{1} / n_{x}^{1}\right)$. Thus, the eigendirection $\Phi_{\sigma}(z)$ profiles obtained for several particle elongations and plane inclinations are illustrated in Fig. 7. First, we obtain that the stress eigendirections are virtually unchanged in the bulk region (see Fig. 7). Moreover, the values of $\Phi_{\sigma}(z)$ do not practically vary when changing the plane inclination (macroscopic shear rate). However, the value of $\Phi_{\sigma}$ notably decreases when increasing particle elongation. Thus, similar to the case of spheres, the stress and strain eigendirections are nonaligned in the shear plane. Interestingly, the angular shift $\Delta \Phi_{\sigma \varepsilon}=\Phi_{\sigma}-\Phi_{\varepsilon}$ increases with particle elongation (inset of Fig. 8), which correlates with the tendency of elongated particles to form an angle between their orientation and the shear 


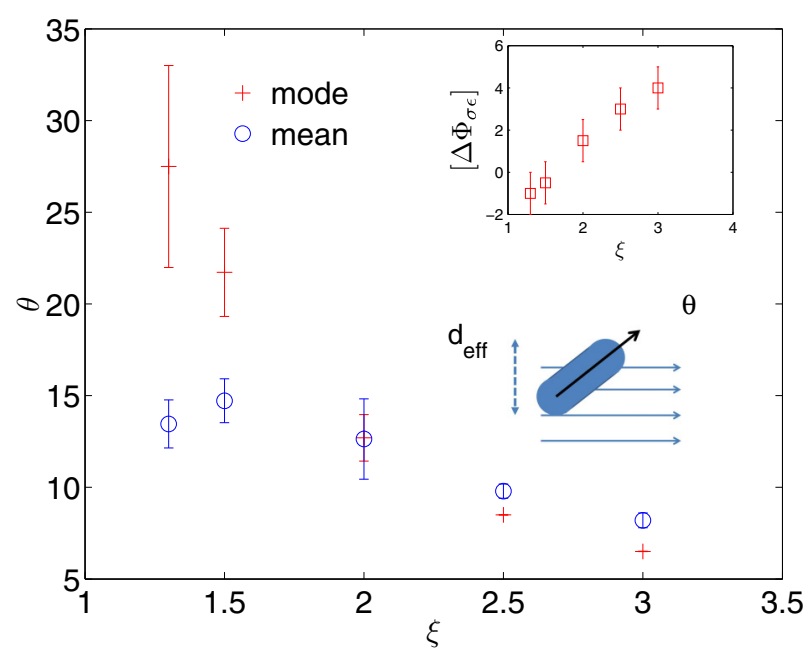

FIG. 8. Numerical results: alignment angle $\theta$, mode, and mean, as functions of particle's aspect ratio $\xi$. The confidential intervals include particles in the bulk region $z \in\left(2 L, h_{m}-2 L\right)$ and several inclinations. The inset shows the angular shift $\Delta \Phi_{\sigma \varepsilon}$ between maximal eigendirection of stress and strain, depending on $\xi$. The sketch illustrates the definition of $d_{\text {eff }}$.

direction. This finding is in accordance with results of Campbell [21], who suggested that in dense ellipsoidal flows, the individual particles choose a preferential orientation to minimize rotation.

\section{Flow rheological response}

Next, we investigate the dependence of the alignment angle $\theta$ on particle elongation $\xi$, as well as its significance in the system micromechanics. For simplicity, this analysis is done only for particles in the bulk region. Figure 8 shows conclusively that the alignment angle, $\theta$ (mean and mode) obtained in the bulk region decreases with particle elongation, for $\xi>1.5$. Similar to sheared systems [21], we find that the variances of the angular distributions (data not shown) are lower for greater $\xi$ and the peaks are better defined, which suggests that particle shape determines the preferred orientation [21]. It is also interesting to note that while the average alignment angle changes considerably (decreases by about $20^{\circ}$ as the aspect ratio increases from 1.3 to 3.0), the shift between the maximal eigendirection of stress and strain is much less.

We go now one step further and examine the significance of particle alignment on the particle layer thickness, which is a crucial parameter in the theoretical formalism of the $\mu(I)$ rheology. To quantify this effect, we define the effective particle size, $d_{\mathrm{eff}}$, as the length of the particle's projection onto the $z$ axis, $d_{\text {eff }}:=2 r+l\left\langle\sin \left(\theta_{\mathrm{p}}\right)\right\rangle$, as illustrated by the inset of Fig. 8, where $\theta_{\mathrm{p}}$ is the most probable alignment angle (mode) represented in Fig. 8. Note that $d_{\text {eff }}$ is a good measure for the thickness of a particle layer, as the flow is dense and thus the mean free path is much lower than the particle size [57]. Thus, the flow of nonspherical particles should be governed by the effective inertial number,

$$
I_{\mathrm{eff}}:=\frac{\dot{\gamma} d_{\mathrm{eff}}}{\sqrt{\sigma_{z z} / \rho_{p}}} .
$$

As we pointed out earlier, the $\mu(I)$ rheology reproduces experimental and numerical observations from a number of setups [7-14]. It has been empirically shown that the macroscopic friction $\mu$ and the density $\rho$ can be expressed as a function of the inertial number $I$ and the expressions read as [2]

$$
\begin{gathered}
\mu(I)=\mu_{1}+\left(\mu_{2}-\mu_{1}\right) \frac{I}{I+I_{o}}, \\
\rho(I)=\rho_{o}-\Delta_{\rho} I,
\end{gathered}
$$



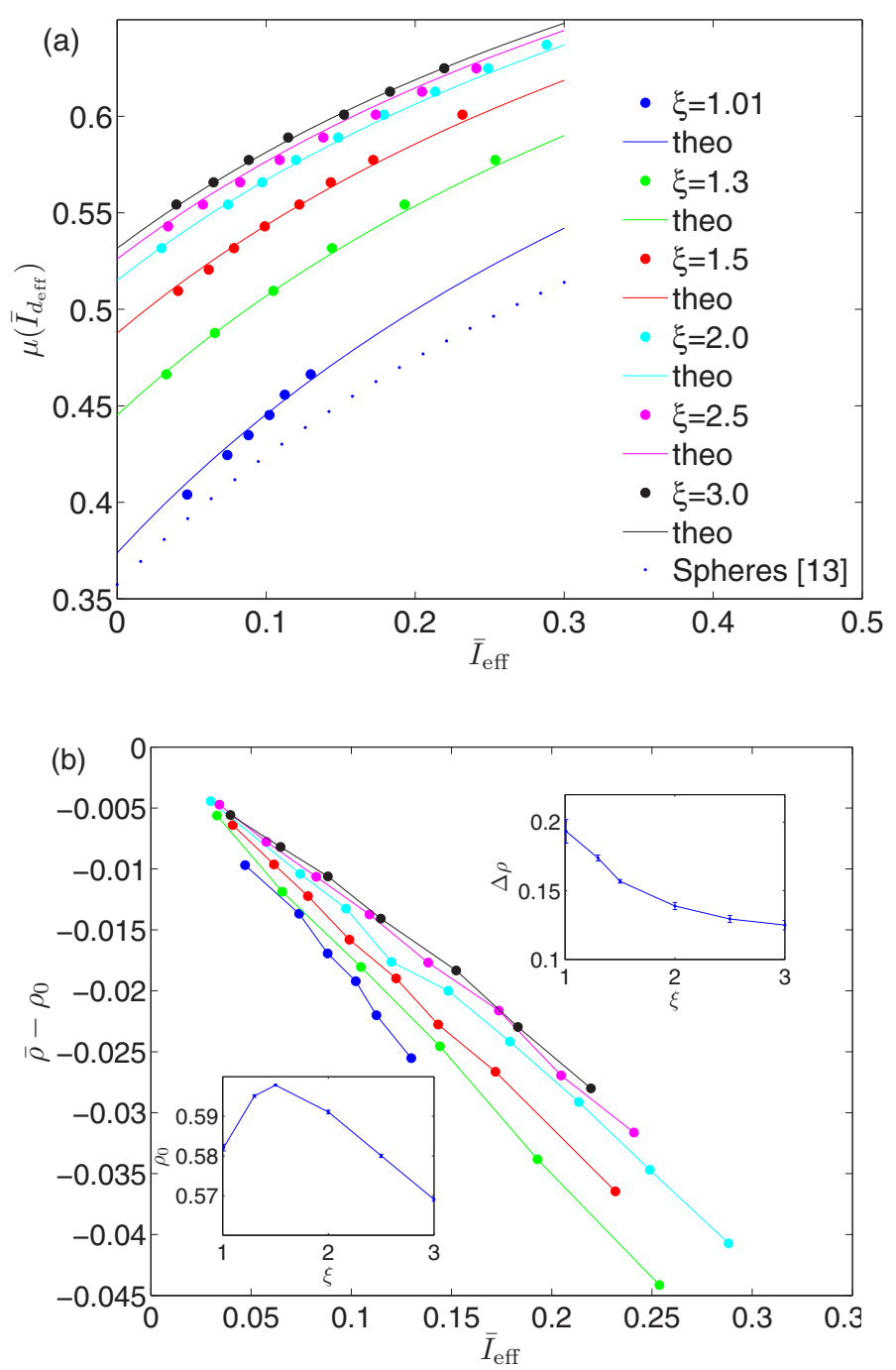

FIG. 9. Numerical results: (a) Depth-averaged friction $\bar{\mu}$ as a function of depth-averaged inertial number, $\bar{I}_{\text {eff }}$. (b) Depth-averaged bulk density from the low-inertia limit $\bar{\rho}-\rho_{0}$, as a function of $\bar{I}_{\text {eff }}$. Insets show the dependence of the bulk density at the static limit $\rho_{0}$ and the slope $\Delta_{\rho}$ on the particle aspect ratio $\xi$.

where $\mu_{1}=\tan \left(\alpha_{c 1}\right)$ and $\mu_{2}=\tan \left(\alpha_{c 2}\right)$. Note that $\alpha_{c 1}$ and $\alpha_{c 2}$ are defined earlier in the text. Besides, $I_{o}$ represents a scaling parameter for the inertial number, $\rho_{o}$ is the bulk density at the static limit, and $\Delta_{\rho}$ is the rate of decrease in density with increasing $I$. These parameters are material dependent [2]. Next, we postulate that for the case of rods the effective inertial number $I=I_{\mathrm{eff}}$, defined in Eq. (5), fully captures the impact of the particle shape, using the same theoretical formalism.

In Fig. 9(a), we plot the depth-averaged macroscopic friction $\bar{\mu}$ against the depth-averaged inertial number $\bar{I}_{\text {eff }}$ for several particle elongations. Remarkably, in all cases the behavior of the internal friction $\bar{\mu}$ agrees very well with Eq. (7), including the measured angle of repose $\alpha_{c 1}(\xi)$, while keeping $\theta_{2}=41.0^{\circ}$ and $I_{o}=0.617$ fixed. Our outcomes indicate that while the shape of the particle is an important factor in dense flows, it is plausible to expect a negligible dependence on $\alpha_{c 2}$, as it accounts for the friction at high-inertia, low-density flows. Also, the bulk friction notably increases as the particle aspect ratio $\xi$ increases, while keeping constant the inertial number $\bar{I}_{\text {eff }}$. Thus, we find 


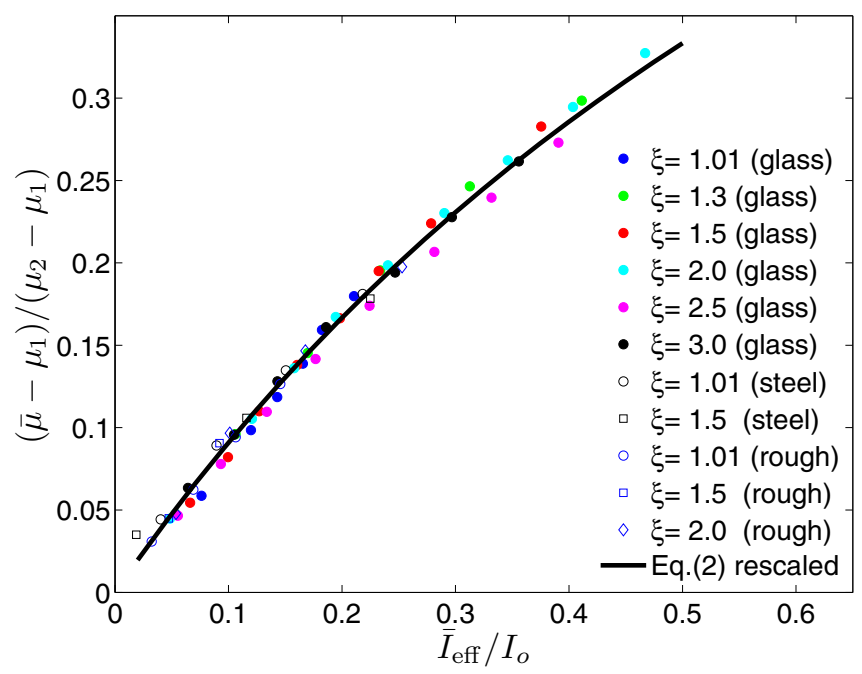

FIG. 10. Numerical results, data collapse obtained when plotting the relative friction versus the depthaveraged inertial number $\bar{I}_{\text {eff }}$. The continuous lines represent Eq. (7). Results for glass and steel particles with different frictions and $g=1 \mathrm{~m} / \mathrm{s}^{2}$ are shown.

that $d_{\text {eff }}$ is an appropriate length scale to define an effective inertial number $I_{\text {eff }}$, which fully captures the impact of the particle shape on the system's rheology.

In order to ensure the validity of our statement, in Fig. 10 we plot the relative friction versus the depth-averaged inertial number $\bar{I}_{\text {eff }}$ in terms of $I_{o}$, which is fixed for all $\xi$. Remarkably, all curves collapse onto a master curve, even including numerical data obtained for different types of materials and particle friction. Hence, results representing different aspect ratios are in excellent agreement with the $\mu(I)$-rheology law, Eq. (7). Note that obtaining the data collapse is achieved with only the critical angle $\alpha_{c 1}(\xi)$ depending on particle elongation. The values of $I_{o}$ and $\alpha_{c 2}$ are kept fixed and thus are considered as material constants. This is achieved by the appropriate selection of the particle's characteristic size $d_{\text {eff }}(\xi)$ when defining $I_{\text {eff }}$. In our case, the careful description of the flow morphology allows obtaining $d_{\text {eff }}(\xi)$ accurately. Thus, the consistency of our results validates the use of the $\mu(I)$ theoretical scheme [7] when describing flows of elongated particles.

As we pointed out earlier, when formulating a granular constitutive model in terms of the inertial number, the behavior of the macroscopic density is also a key point. Thus, we examine the relation between depth-averaged macroscopic density $\bar{\rho}$ and $\bar{I}_{\text {eff }}$, for various particle aspect ratios $\xi$ [see results shown in Fig. 9(b)]. We find that the decrease in the macroscopic density $\bar{\rho}$ correlates with the decrease of inertial number, and thus it can be fitted to Eq. (7). However, we observe that the particle elongation $\xi$ significantly affects the rate of decrease, $\Delta_{\rho}$ [inset of Fig. 9(b)].

Moreover, when plotting the estimation of the low inertia limit $\rho_{0}$ as a function of $\xi$ [inset of Fig. 9(b)], we find nonmonotonic behavior, similar to the $\xi$ dependence of the random close packing density [61]. Note that increasing $\xi$ from the spherical limit leads to increase in the number of contacts and, as a result, more dense structures are obtained [61]. For $\xi>1.5$, however, $\rho_{0}(\xi)$ decreases with increasing elongation, which is also consistent with previous experimental findings in 2D [62] and $3 \mathrm{D}$ [61] packings. In that regime, the decrease in the density is explained by strong excluded-volume effects, induced by the orientation disorder [61]. In Fig. 9(b), we plot the difference between the depth-averaged density, $\bar{\rho}$, and $\rho_{0}$ as a function of the depth-averaged effective inertial number, $\bar{I}_{\text {eff }}$.

\section{CONCLUSIONS AND REMARKS}

In conclusion, a granular flow of nonspherical particles down an inclined plane is investigated in detail. First, the flow morphology is characterized, exploring the particle orientation $\theta$ and its relation 
to the direction and magnitude of the shear rate $\dot{\gamma}$. As expected, particle orientation in the bulk varies distinctly from the behavior at the surface. In the bulk, the orientation of particles is slightly inclined with respect to the flow, and this behavior is independent of the shear rate. At the surface, however, the particles move in more dilute conditions, and we find that $\theta_{\mathrm{av}}$ decreases with increasing $\dot{\gamma}$. This trend is similar to the behavior of a single Brownian rod embedded in a sheared liquid [32,33]. Second, we explore the impact of particle shape on the system's rheological response by varying the particle aspect ratio and the plane inclination. A careful analysis of the system's characteristic time and length scales reveals that the particle size perpendicular to the flow direction is an appropriate length scale to define an effective inertial number $I_{\mathrm{eff}}$. Our outcomes indicate conclusively that $I_{\mathrm{eff}}$ fully captures the impact of the particle shape, in the $\mu(I)$ theoretical formalism [7]. Furthermore, similarly to the case of spheres, density and friction are well-defined functions of $I_{\text {eff }}$. Hence, we quantify and explain the dependence of the rheological parameters on particle aspect ratio, based on the micromechanical details. We expect that the present findings will help to better characterize more complex flows, e.g., in funnels or silos. Moreover, our outcomes raise new questions about whether this kind effective inertial number scheme captures the effect of the particle shape in systems composed of complex elongated shapes. These questions will be addressed in future works.

\section{ACKNOWLEDGMENTS}

The Spanish MINECO (FIS2017-84631-P MINECO/AEI/FEDER, UE Projects), the German DFG LU 450/10 (SPP PiKo), and the Hungarian NKFIH (K 116036) projects supported this work.

[1] C. S. Campbell, Granular material flows: An overview, Powder. Technol. 162, 208 (2006).

[2] P. Jop, Y. Forterre, and O. Pouliquen, A constitutive law for dense granular flows, Nature (London) 441, 727 (2006).

[3] Y. Jiang and M. Liu, Granular solid hydrodynamics, Granul. Matter 11, 139 (2009).

[4] K. Kamrin and G. Koval, Nonlocal Constitutive Relation for Steady Granular Flow, Phys. Rev. Lett. 108, 178301 (2012).

[5] Q. Zhang and K. Kamrin, Microscopic Description of the Granular Fluidity Field in Nonlocal Flow Modeling, Phys. Rev. Lett. 118, 058001 (2017).

[6] T. Barker, D. G. Schaeffer, M. Shearer, and J. M. N. T. Gray, Well-posed continuum equations for granular flow with compressibility and (i)-rheology, Proc. R. Soc. London, Ser. A 473, 20160846 (2017).

[7] GDR-MiDi, On dense granular flows, Eur. Phys. J. E. 14, 341 (2004).

[8] F. da Cruz, S. Emam, M. Prochnow, J. N. Roux, and F. Chevoir, Rheophysics of dense granular materials: Discrete simulation of plane shear flows, Phys. Rev. E 72, 021309 (2005).

[9] T. Hatano, Power-law friction in closely packed granular materials, Phys. Rev. E 75, 060301 (2007).

[10] T. Börzsönyi, R. E. Ecke, and J. N. McElwaine, Patterns in Flowing Sand: Understanding the Physics of Granular Flow, Phys. Rev. Lett. 103, 178302 (2009).

[11] E. Azéma and F. Radjaï, Internal Structure of Inertial Granular Flows, Phys. Rev. Lett. 112, 078001 (2014).

[12] M. Tankeo, P. Richard, and E. Canot, Analytical solution of the $\mu(i)-$ rheology for fully developed granular flows in simple configurations, Granul. Matter 15, 881 (2013).

[13] T. Weinhart, A. R. Thornton, S. Luding, and O. Bokhove, Closure relations for shallow granular flows from particle simulations, Granul. Matter 14, 531 (2012).

[14] A. Fall, G. Ovarlez, D. Hautemayou, C. Méziére, J.-N. Roux, and F. Chevoir, Dry granular flows: Rheological measurements of the $\mu$ (i)-rheology, J. Rheol. 59, 1065 (2015).

[15] R. A. Bagnold, Experiments on a gravity-free dispersion of large solid spheres in a Newtonian fluid under shear, Proc. Roy. Soc. London, Ser. A 225, 49 (1954). 
[16] L. E. Silbert, D. Ertas, G. S. Grest, T. C. Halsey, D. Levine, and S. J. Plimpton, Granular flow down an inclined plane: Bagnold scaling and rheology, Phys. Rev. E 64, 051302 (2001).

[17] R. Delannay, M. Louge, P. Richard, N. Taberlet, and A. Valance, Towards a theoretical picture of dense granular flows down inclines, Nat. Mater. 6, 99 (2007).

[18] H. Katsuragi, A. R. Abate, and D. J. Durian, Jamming and growth of dynamical heterogeneities versus depth for granular heap flow, Soft Matter 6, 3023 (2010).

[19] M.Y. Louge, A. Valance, P. Lancelot, R. Delannay, and O. Artiéres, Granular flows on a dissipative base, Phys. Rev. E 92, 022204 (2015).

[20] K. A. Reddy, V. Kumaran, and J. Talbot, Orientational ordering in sheared inelastic dumbbells, Phys. Rev. E 80, 031304 (2009).

[21] C. S. Campbell, Elastic granular flows of ellipsoidal particles, Phys. Fluids 23, 013306 (2011).

[22] T. Börzsönyi, B. Szabó, G. Törös, S. Wegner, J. Török, E. Somfai, T. Bien, and R. Stannarius, Orientational Order and Alignment of Elongated Particles Induced by Shear, Phys. Rev. Lett. 108, 228302 (2012).

[23] T. Börzsönyi, B. Szabó, S. Wegner, K. Harth, J. Török, E. Somfai, T. Bien, and R. Stannarius, Shear-induced alignment and dynamics of elongated granular particles, Phys. Rev. E 86, 051304 (2012).

[24] F. Villemot and J. Talbot, Homogeneous cooling of hard ellipsoids, Granul. Matter 14, 91 (2012).

[25] K. Harth, U. Kornek, T. Trittel, U. Strachauer, S. Höme, K. Will, and R. Stannarius, Granular Gases of Rod-Shaped Grains in Microgravity, Phys. Rev. Lett. 110, 144102 (2013).

[26] Y. Guo, C. Wassgren, W. Ketterhagen, B. Hancock, B. James, and J. Curtis, A numerical study of granular shear flows of rod-like particles using the discrete element method, J. Fluid Mech. 713, 1 (2012).

[27] Y. Guo, C. Wassgren, B. Hancock, W. Ketterhagen, and J. Curtis, Granular shear flows of flat disks and elongated rods without and with friction, Phys. Fluids 25, 063304 (2013).

[28] H. Pourtavakoli, E. J. R. Parteli, and T. Pöschel, Granular dampers: Does particle shape matter? New J. Phys. 18, 073049 (2016).

[29] T. Trittel, K. Harth, and R. Stannarius, Mechanical excitation of rodlike particles by a vibrating plate, Phys. Rev. E 95, 062904 (2017).

[30] K. Harth, T. Trittel, S. Wegner, and R. Stannarius, Free Cooling of a Granular Gas of Rodlike Particles in Microgravity, Phys. Rev. Lett. 120, 214301 (2018).

[31] E. J. Hinch and L. G. Leal, The effect of Brownian motion on the rheological properties of a suspension of non-spherical particles, J. Fluid Mech. 52, 683 (1972).

[32] J. Vermant, H. Yang, and G. G. Fuller, Rheooptical determination of aspect ratio and polydispersity of nonspherical particles, AIChE 47, 790 (2001).

[33] J. K. Dhont and W. J. Briels, Rod-like Brownian particles in shear flow, in Soft Matter (Wiley-VCH, Berlin, 2007), p. 147.

[34] G. Lu, J. R. Third, and C. R. Muller, Discrete element models for non-spherical particle systems: From theoretical developments to applications, Chem. Eng. Sci. 127, 425 (2015).

[35] C. M. Wensrich and A. Katterfeld, Rolling friction as a technique for modeling particle shape in DEM, Powder. Technol. 217, 409 (2012).

[36] J. P. Latham, A. Munjiza, X. Garcia, J. Xiang, and R. Guises, Three-dimensional particle shape acquisition and use of shape library for DEM and FEM/DEM simulation, Miner. Eng. 21, 797 (2008).

[37] M. A. Hopkins, A. V. Potapov, and C. S. Campbell, A two-dimensional dynamic simulation of solid fracture part I: Description of the model, Int. J. Mod. Phys. C 6, 371 (1995).

[38] E. Azéma, F. Radjai, and F. Dubois, Packings of irregular polyhedral particles: Strength, structure, and effects of angularity, Phys. Rev. E 87, 062203 (2013).

[39] M. Boton, E. Azéma, N. Estrada, F. Radjaï, and A. Lizcano, Quasistatic rheology and microstructural description of sheared granular materials composed of platy particles, Phys. Rev. E 87, 032206 (2013).

[40] T. Kanzaki, M. Acevedo, I. Zuriguel, I. Pagonabarraga, D. Maza, and R. C. Hidalgo, Stress distribution of faceted particles in a silo after its partial discharge, Eur. Phys. J. E. 34, 133 (2011).

[41] G. Lu, J. R. Third, and C. R. Müller, Effect of particle shape on domino wave propagation: A perspective from 3D, anisotropic discrete element simulations, Granul. Matter 16, 107 (2014).

[42] S. M. Rubio-Largo, F. Alonso-Marroquín, T. Weinhart, S. Luding, and R. C. Hidalgo, Homogeneous cooling state of frictionless rod particles, Phys. A (Amsterdam, Neth.) 443, 477 (2016). 
[43] K. Van der Werf, W. Jin, M. D. Shattuck, and C. S. O'Hern, Hypostatic jammed packings of frictionless nonspherical particles, Phys. Rev. E 97, 012909 (2018).

[44] J. E. Andrade, K. W. Lim, C. F. Avila, and I. Vlahinić, Granular element method for computational particle mechanics, Comput. Methods Appl. Mech. Eng. 241-244, 262 (2012).

[45] R. Kawamoto, E. Andø, G. Viggiani, and J. E. Andrade, Level set discrete element method for threedimensional computations with triaxial case study, J. Mech. Phys. Solids 91, 1 (2016).

[46] S. Mandal and D. V. Khakhar, A study of the rheology of planar granular flow of dumbbells using discrete element method simulations, Phys. Fluids 28, 103301 (2016).

[47] S. Mandal and D. V. Khakhar, A study of the rheology and micro-structure of dumbbells in shear geometries, Phys. Fluids. 30, 103303 (2018).

[48] C. Kloss, C. Goniva, A. Hager, S. Amberger, and S. Pirker, Models, algorithms, and validation for open source DEM and CFD-DEM, Progr. Comput. Fluid. Dynam. Int. J. 12, 140 (2012).

[49] R. Guises, J. Xiang, J. P. Latham, and A. Munjiza, Granular packing: Numerical simulation and the characterisation of the effect of particle shape, Granul. Matter 11, 281 (2009).

[50] E. Somfai, D. B. Nagy, P. Claudin, A. Favier, D. Kálmán, and T. Börzsönyi, Effective friction of granular flows made of non-spherical particles, EPJ Web Conf. 140, 03062 (2017).

[51] D. B. Nagy, P. Claudin, T. Börzsönyi, and E. Somfai, Rheology of dense granular flows for elongated particles, Phys. Rev. E 96, 062903 (2017).

[52] R. C. Hidalgo, S. M. Rubio-Largo, F. Alonso-Marroquin, and T. Weinhart, Non-spherical granular flows down inclined chutes, EPJ Web Conf. 140, 03007 (2017).

[53] T. Pöschel and T. Schwager, Computational Granular Dynamics (Springer-Verlag, Berlin, 2005).

[54] M. Babic, Average balance equations for granular materials, Int. J. Eng. Sci. 35, 523 (1997).

[55] I. Goldhirsch, Stress, stress asymmetry, and couple stress: From discrete particles to continuous fields, Granul. Matter 12, 239 (2010).

[56] R. Artoni and P. Richard, Average balance equations, scale dependence, and energy cascade for granular materials, Phys. Rev. E 91, 032202 (2015).

[57] T. Weinhart, R. Hartkamp, A. R. Thornton, and S. Luding, Coarse-grained local and objective continuum description of three-dimensional granular flows down an inclined surface, Phys. Fluids 25, 070605 (2013).

[58] J. A. C. Gallas and S. Sokolowski, Grain non-sphericity effects on the angle of repose of granular material, Int. J Mod. Phys. B 7, 2037 (1993).

[59] H. G. Matuttis, S. Luding, and H. J. Herrmann, Discrete element simulations of dense packings and heaps made of spherical and non-spherical particles, Powder. Technol. 109, 278 (2000).

[60] Z. Y. Zhou, R. P. Zou, D. Pinson, and A. B. Yu, Angle of repose and stress distribution of sand piles formed with ellipsoidal particles, Granul. Matter 16, 695 (2014).

[61] A. Donev, I. Cisse, D. Sachs, E. A. Variano, F. H. Stillinger, R. Connelly, S. Torquato, and P. M. Chaikin, Improving the density of jammed disordered packings using ellipsoids, Science 303, 990 (2004).

[62] R. C. Hidalgo, I. Zuriguel, D. Maza, and I. Pagonabarraga, Granular packings of elongated faceted particles deposited under gravity, J. Stat. Mech. (2010) P06025. 